\title{
An introduction to this special section: Full-waveform inversion and the way forward
}

\author{
Antolne Guitton, San Mateo, USA
}

Tario AlkhalfaA, Thuwal, Saudi Arabia

$\mathrm{L}$ ost in the endless valleys and hills of the full-waveform inversion (FWI) misfit functional, we tend to stop and wonder: Are we heading in the right direction? Are we in the right valley? Or within a bigger context, is FWI the way to go? The practice of updating an Earth model and generating synthetic data from it that we can compare to the field data is an appealing concept. If the two data sets (the modeled and field) match, using some measure of misfit, we might have found a good Earth model. This process depends on our ability to fully replicate (simulate) the physics of wave propagation inside the Earth. In principle, our field data carry information from every point in the Earth, but these data are also constrained by the geometry of seismic acquisition.

Therefore we attempt, in a straightforward fashion, to duplicate the experiment in the field with a computer simulation (Tarantola, 1984). We are usually ignorant of the source signature, so we try to invert for it. Given the computing resources available and the data to be matched, we approximate the physics of wave propagation as well as we can and hope data preconditioning allows us to get away with it. However, the critical information that we need to simulate wave propagation, and the reason why the process is called inversion, are the medium parameters (the elastic coefficients or their practical simplification in acoustic media) and the P-wave velocity. Thus, we assume that the majority of the difference between the simulated data and the field data is attributable to having the wrong model parameters and, thus, we use the information contained in the difference (i.e., data misfit) to improve them.

Therefore, FWI describes the process of fitting our synthetic data to field data by updating the appropriate medium parameters in order to optimize this fit. The updating process is open to many possibilities, from Taylor-series-induced updates (Tarantola, 1984) to model distribution probability and stochastic methods (Sen and Stoffa, 1992; Mallick, 1999). The most affordable, and therefore popular, process is the gradient-based technique: the model is updated iteratively by following a local descent direction (the gradient). Surprisingly, this descent direction is mathematically equivalent to a reverse time migration (Lailly, 1983). This simple yet powerful fact explains why FWI has re-emerged in seismic processing: recent advances in computing software and hardware, which make RTM feasible for large 3D projects, can be used to speed up FWI. It should be noted that a low-frequency run of FWI $(<10 \mathrm{~Hz})$, including all iterations, can cost less than a single high-frequency RTM run $(>50 \mathrm{~Hz})$. Despite a multitude of updating options, the objective of FWI stays admirable, straightforward, and easy to understand: matching our simulated data to the field data.

The biggest FWI implementation challenge is the significant nonlinearity in the objective function, because of the sinusoidal nature of seismic wavefields and Earth model complexity. A gradient-based update of the velocity model requires an initial velocity field that produces synthetic data that are within a half a cycle of the field data, resulting in a misfit located within the basin of attraction of the true solution of the objective function. Clearly, the size of this basin of attraction and the length of the half cycle increase at low frequencies. However, we rarely acquire field data with frequencies low enough that we can start FWI from a crude initial velocity model. Stochastic approaches would not suffer from this local minimum issue and would converge to the global minimum, but exhaustively exploring the model space is still far beyond current computing capabilities.

In summary, some of the main obstacles to a successful FWI (or proper convergence) are the following:

- The strong nonlinearity (i.e., cycle skipping) of the problem, which requires accurate starting models when gradient-based methods are used to update the model space.

- The limited/poor illumination of the subsurface because of the surface seismic acquisition geometries, which requires regularization of the inversion.

- The anelastic and anisotropic nature of the Earth, which requires more accurate simulations, better data conditioning, and (most importantly) better multiparameter inversion strategies.

- The computational cost of FWI, which requires at least three wavefield simulations at each of 10-50 iterations.

This special section comprises 12 articles from leading researchers in the field, from both academia and industry, discussing subjects ranging from multiparameter inversion, combining FWI with migration velocity analysis, improved efficiency and case studies. As seen in Figure 1, the number of SEG publications related to FWI has exploded since 2005, covering all aspects of the method. To help organize this rapidly growing topic, we selected four themes related to current practices and research directions in FWI. Admittedly, this classification is arbitrary as many articles could belong to more than one theme. The first three themes cover theoretical aspects of FWI, with some synthetic examples and case studies included, while the last one focuses on case studies only. This classification represents the status of FWI, transitioning slowly but surely out of the academic world into the unforgiving realm of field data.

The first theme describes methodologies and examples of multiparameter FWI. Here, the authors present results and techniques to derive not only P-wave velocity models, but also anisotropic parameters, attenuation, density, etc. Plessix et al. show how intermediate-to-long wavelengths of the velocity 
model can be retrieved with acoustic FWI incorporating long offsets and low-frequency $(2 \mathrm{~Hz})$ data, and by focusing on diving waves. Inverting for anisotropic parameters and/or density is also crucial for a better kinematic and amplitude fit of the data. Operto et al. tackle the difficult task of finding the best parameterization for multiparameter acoustic and elastic FWI. In this article, they propose different combinations of parameters suited for FWI by analyzing radiation patterns. Radiation patterns show the amplitude of the partial derivative wavefield with scattering angle for a model-parameter perturbation, keeping the other parameters fixed. This article presents theoretical as well as practical aspects of multiparameter FWI; their findings are illustrated on synthetic as well as field data. Finally, Wang et al. present a regularization technique to constrain the velocity model with well data. They also show, with field data, how acoustic FWI can be used to invert for VTI anisotropic parameters as well as attenuation.

The second theme presents research directions where the authors try to combine the robustness of existing wave-equation-migration velocity analysis (WEMVA) techniques with FWI. This combination makes sense: unlike FWI, WEMVA methods do not suffer from the local minima issue and, thus, can start from a relatively poor initial model, while yielding improved low-resolution models needed for FWI. This section presents two relatively theoretical articles investigating this possibility. Diaz et al. show how cascading image and data-domain wavefield tomography might eliminate the need for low frequencies in the data. Biondi and Almomin improve the convergence of FWI by extending the velocity model along the time-lag axis.

The third theme deals with schemes in the FWI implementation tailored to improve efficiency. Herrmann et al. describe a technique that adaptively selects a subset of the shot gathers at each iteration by stochastic optimization. This method builds more accurate data misfits and gradients as the model improves, thus saving computational time. Contrary to all other articles in this special section, Shin et al. run FWI in the Laplace domain. A Laplace-domain wavefield is equivalent to the zero frequency of a damped wavefield. Working with the zero frequency of a damped wavefield, the objective function defined in the Laplace domain has fewer local minima and the velocity model contains long wavelengths only. Therefore, the Laplace domain can be used to derive starting models for FWI. For those interested in the details of this method, Ha and Shin (2013) provide valuable insights into the Laplace domain.

Finally, the fourth theme introduces FWI case studies. These articles are probably most relevant to practitioners and readers trying to convince themselves that the "dark art" of FWI really works. Bansal et al. show how large-scale 3D FWI projects are made possible by using shot-encoding strategies: shots are encoded and summed together before inversion to minimize computational costs. Ratcliffe et al. present FWI results using data acquired with variable-depth streamers. This geometry enables lower frequencies to be recorded. These low frequencies help in recovering shallow channels in the North Sea. Another acquisition approach that may serve FWI well is presented in Vigh et al. Here, the dual-coil multivessel method helps acquire long offsets (potentially up to $29 \mathrm{~km}$ !) These large offsets help FWI resolve some of the missing intermediate wavelengths of the velocity model, necessary to connect the initial smooth model to the desired high resolution model we are expecting as an FWI deliverable. A complementary study analyzing the effect of long offsets on FWI, as well as their relationship with the long-wavelengths of the velocity model, is provided by Liu et al. Finally, Kelly et al. show how both reflections and refractions can be used to derive velocity models. Using reflections in FWI is not a simple task as they contain both velocity and density information that is difficult to separate (look at the article from Operto et al. in this section to convince yourself).

Full-waveform inversion was promised to be the final and ultimate solution to the Earth resolution and imaging objective: we are now waiting for this promise to materialize or, more pragmatically, learning what can be expected from this re-emerging method in realistic exploration settings. FEE

\section{References}

Ha, W., and C. Shin, 2013, Why do Laplace-domain waveform inversions yield long-wavelength results?: Geophysics, 78, no. 4, R167R173, http://dx.doi.org/10.1190/geo2012-0365.1.

Lailly, P., 1983, The seismic inverse problem as a sequence of beforestack migrations in J. Bednar, ed., Conference on inverse scattering, theory and applications, Society for Industrial and Applied Mathematics, 206-220.

Mallick, S., 1999, Some practical aspects of prestack waveform inversion using a genetic algorithm: An example from the east Texas Woodbine gas sand: Geophysics, 64, no. 2, 326-336, http:// dx.doi.org/10.1190/1.1444538.

Sen, M. K. and P. L. Stoffa, 1992, Rapid sampling of model space using genetic algorithms: Examples from seismic waveform inversion: Geophysical Journal International, 108, no. 1, 281-292, http://dx.doi.org/10.1111/j.1365-246X.1992.tb00857.x.

Tarantola, A., 1984, Inversion of seismic reflection data in the acoustic approximation: Geophysics, 49, no. 8, 1259-1266, http://dx.doi. org/10.1190/1.1441754.

\section{Corresponding author: aguitton@gmail.com}

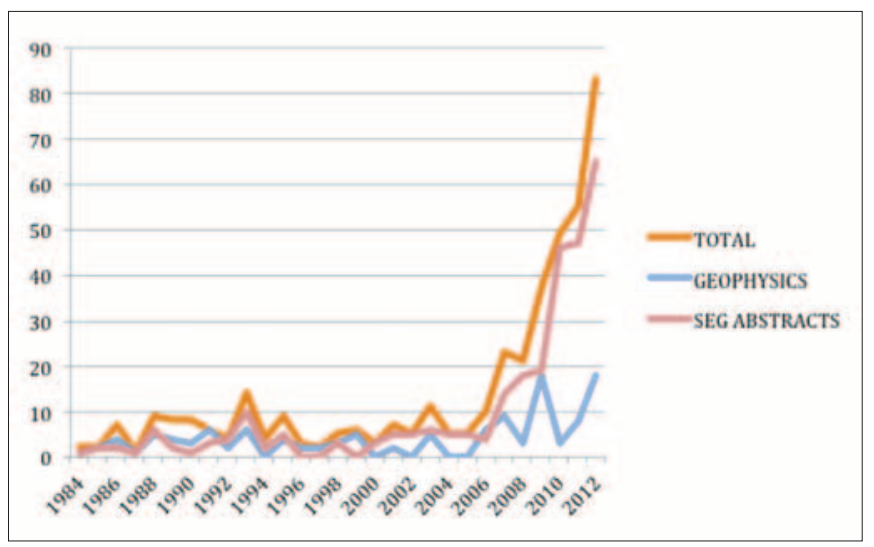

Figure 1. Approximate number of SEG publications with the words "fullwaveform inversion" or "waveform inversion" or "waveform tomography" in the title. A significant increase of publications is visible after 2005 (corresponding to the rebirth of RTM as a viable imaging technique). 